\title{
Short Note on Space Wind Powered by Disorder: Dark Energy
}

\author{
Mesut KAVAK*
}

\begin{abstract}
After midnight just before sleeping, I noticed in my bed, that free space itself can cause a parachute effect on the moving bodies especially the bodies move in low gravitational fields like in Pioneer Anomaly. Because of this reason, while speed of a satellite is decreasing, speed of another one which spins around the world on different axis can increase; a satellite wandering in interstellar medium can speed up as also it can slow down; low frequency light and high frequency light behave differently; galaxies have lower mass can spin faster since mutual gravitation is not only option. These are only a few examples.
\end{abstract}

\section{Introduction}

Because of uncertainty, matter could not gain infinite energy since the work done for creating matter was not done by infinite energy, since work done is equal to kinetic energy. It emerges in a time interval and at a limited frequency according to the creation work's speed. There is only one work to create all the universe because of an absolute entanglement, and thus any matter in this universe gains energy due to the speed of the work done. Matter uses the same space during any act; so it cannot go faster than light since already it moves at speed of light because of the motion which is caused by the work done to create.

\section{Natural holding capacity of free space}

Emergence in this way renders impossible to move of bodies linear manner since there is no middle point for any force applied because of uncertainty. Any short interval has shorter interval to infinite small part of matter at the same time, even before you measure it. By this way, flexible collision is not possible and thus information is conserved forever since there is not going to be certain destruction during collisions. The amounts get smaller until infinite small amount but it never disappears. Because of this reason, motion can only emerge in circular ways. Actually we can call it a motion which closes a curve; because it is not a perfect circle. Even actually we should call it a motion which does not close a curve because of uncertainty. It is only assumed to calculate it. It does not close a curve; because it is just a part of infinite information. All infinite information is one piece and closes a curve since you cannot add in or detract from infinity as anything is element of infinity. They already existed. It is deterministic. It only appears being in a cause and effect relationship with infinite information.

These also mean, that any mass or energy is only distributed mass or energy because of uncertainty; but the problem is it, that which stringency, frequency, density does matter have on free space because of its existence? What is the reference? Namely, what is the amount of emptiness. As entire universe emerges at a limited frequency, it is not constant at every point of its free space. Empty space which has no particle or wave also has a frequency. Before emergence of a wave, there must exist free space firstly since particles emerge over this space as waves; so particles cannot have higher frequency than this frequency of free space; because there shall no place to emerge after this limit. You must increase the creation frequency and thus light speed or creation speed of the entire universe and thus of the single work done.

As any mass or energy can only be counted as distributed mass or energy because of uncertainty, being $f$ is total frequency of the universe, $V$ is volume of the universe, natural frequency holding capacity of free space becomes $C=f / V$ for 1 cubic meter. Space cannot hold higher frequency than this in 1 cubic meter naturally whatever the mass scatter is in this volume. If it exists, then space is going to directly be distended. This place then becomes more disordered, and then wants to be fixed by distribution on space, on a wider area because of incompressibility property of matter which is because of limited light speed. Denser space is more disordered; because you need more speed to create denser area since application time and thus speed of the force applied are going to change. Actually more speed is only required after you compressed all the mass of the universe to the old position, then you need more speed and thus the new universe is going to have more energy because of increased speed of the work done since the work done is equal to kinetic energy. Even so you are going to converge to this condition for the densities in the focal points of the universe by some amounts due to density since a stress is going to emerge although you did not compress all the mass of the universe. During this process, for creating denser area with a fixed speed, only disorder will increase as long as you create more and more denser area. If you create a lower density area, it wants to be fixed by an opposite low force; but if you create denser area, the opposite force will be bigger. Also gravity works over this principle. Each gravitational wave creates a potential difference, and when they intersected, the space moves according to the condition that also a repulsion is possible. As matter emerge over this sliding space, also matter moves. Light speed is a gravitational acceleration but an acceleration limit since gravity is the single source of motion; because light always falls during its movement in free space even if is not in an external gravitational field at that time. If you create a potential in space as a wave, it does translational motion in the way of lower density more ordered place which is the movement way, and its extinguishedness time is very long.

This properties may be effective in red giants. If there is not going to be enough gravity which holds mass at the expense of space distension since space can be bended, they cannot prevent inflation. If freely moving alone bodies in medium has low gravitational effects are handled, they are also extremely affected since they have no external supportive gravitational force. Even if you think, that there is only 1 particle in the universe, because of its existence or inner space creation motion which is not observational that actually is the same with observational outer space motion, it is going to move according to spin of the universe; because to be one of them before or after of the same $F t$ work as $+F t$ and $-F t$ changes displacement. According to initial movement way of the universe, the particle is going to move by acceleration since it experiences constant potential difference over time on space because of emergence frequency of free space. It always seeks an ordered area but it cannot find. It never stops.

\section{Conclusion}

As the result, free space has a frequency holding capacity for each cubic meter; so initial velocities of moving bodies 
directly affect own observational outer space constant speed. How many cubic meters they draw per second, this is important. Also their mass and density are important for this drawing. The resistance they experience changes according to these properties. It means, that also different densities of the same frequency light or different frequency lights have different behavior on space. It is going to be misleading if you make the same calculation for different upcoming lights of different size stars. Even red-shift amount is different for more intense light or lower density light or different frequency lights.

Spin of the universe is also pretty important; because as it can cause a deflection on moving bodies, also can cause a resistance or acceleration.

I had derived some properties of the universe over light speed and centrifugal force at the beginning of time, if you assume that the motion is perfect circle that is not actually and there is going to be some differences, such as radius or volume. Additionally I used a little different calculation for Planck constant, Planck length and mass of photon. The results will change in some amounts which are not so different. Holding capacity $C$ for 1 cubic meter is equal to the following formula over this calculations,

$$
C=\frac{3 c \sqrt{2 \pi c G}}{4 \pi G^{2}} \text { (Hertz) }
$$

where $c$ is the speed of light and $G$ is gravitational constant It is nearly $5.7 \cdot 10^{28} \mathrm{Hertz}$. Being $h f=m_{e} c^{2}$ where $m_{e}$ is mass of an electron, $h$ is Planck constant and $f$ is frequency of electron and $c$ is the speed of light; it means, that electron has nearly $1.22 \cdot 10^{20} \mathrm{Hertz}$ frequency. It means, that $1 \mathrm{cu}-$ bic meter space can hold only nearly $5 \cdot 10^{8}$ times electrons. It means, that can hold nearly $d_{s}=4.5 \cdot 10^{-22} \mathrm{~kg} / \mathrm{m}^{3}$ mass Over lifting force of liquids equation of $F_{l}=V_{s} \cdot d_{l} \cdot g$ where $F_{l}$ is lifting force, $V_{s}$ is sinking volume and $d_{l}$ is density of the liquid, $g$ is gravitational acceleration that is equal to light speed for 1 second since gravity is the main and single reason of motion as I proved over Heisenberg's uncertainty equation $\Delta x \Delta p \geq h / 2$ since the equation interprets change in gravitational potential, it becomes

$$
m a_{o}=V_{s} \cdot 1.35 \cdot 10^{-13}
$$

over $m a_{o}=V_{s} \cdot d_{s} \cdot c$, where $F_{l}=m a_{o}, m$ is mass of an object such as vehicle in free space far enough from a gravitational field, $a_{o}$ is observational outer space acceleration different than the gravitational formation speed since outer space and inner space motion are accepted and emerge together by using the same space at the same time that when an object moves from A to B it means emergence motion moved, $c$ is the speed of light as gravitational acceleration here for $1 \mathrm{sec}$ ond over $\mathrm{v}=$ at since it is the source of emergence speed as gravity for any wave or particle.

These calculations are for macro cosmos to determine a reference; so you cannot say mass scatter is not important in 1 cubic meter for micro cosmos. In the same manner, you must calculate each cubic meter of an object separately. Namely, you must use aerodynamics. For example, while one wing of a satellite is lifting the vehicle, the other one can leave it. You must calculate it according to geometry placed in each cubic meter of the existent outer space object.

\section{Warning}

As a result, for 1 ton object which has 10 cubic meters volume, acceleration or deceleration according to spin becomes

$$
a_{o}=1.35 \cdot 10^{-15} \mathrm{~m} / \mathrm{s}^{2}
$$

Alright; but we did not include initial velocity since it draws more volume according to this speed. If you assume, that this object moves at $10 \mathrm{~km}$ per second, for $V_{s} \cdot 10.000$, it becomes

$$
a_{o}=1.35 \cdot 10^{-11} \mathrm{~m} / \mathrm{s}^{2}
$$

This may explain the pioneer anomaly, and also seems galaxies have lower mass magnitude can turn at higher speeds than heavier ones, that is actually the same with sail effect here. Here electron is only a reference. You can use any particle to determine it. This will not change anything.

To experience better experiments such as determining the spin of the universe maybe by length contraction amounts or by the deflection and deceleration amounts, for reaching the light speed experiments, we need a new thrust source which can work in a closed environment that maybe even under a seat or after that by changing on a wall in a space vehicle. As producing thrust, as energy is not independent of force, it can turn into force. To realize this, Eq. (5) or if a blade is used instead of a marble placed at the top of a wire, nearly $F / 2$ centrifugal force is perfect closed system thrust engine potential as independent of mass of air or water without opposite torque, and it can store huge amount of energy by a small energy amount when it is required by some transmission, that the work done is only done against frictions at that time.

$$
F=\frac{m v^{2} \sin (\alpha)}{r}
$$

In classical propeller systems, you must hold on air to produce thrust by propeller blades; so the mass for the energy of the work done is directly must be the vehicle which is aimed to move; but by the above stated method, you can only calculate the mass of a propeller. It can produce thrust as much as tensile strength of the used materials for the propeller and the blades. Just use it as double if you do not want to use an apparatus like in the butt propeller of helicopters because of conservation of momentum. Also if it is connected around a rotating wheel, you can produce torque for any machine. This engine should be used by free energy generating methods.

Universe gets its volume due to centrifugal force; but according to emerging particle amount, the volume changes. If particles evaporates in the other name they experience decrease in their vibration in accordance with entropy, then volume increases. This seems like the universe is expanding but not. Also there is time differences along centrifugal way; thus also this causes motion and again can be seen like expanding [1].

\section{Warning}

\section{Acknowledgment}

Above stated things was done over my papers [1,2]. I did not explain and calculate them again because a little annoying and long. There are some calculations such as gravitational attraction and repulsion; frequency, mass, energy and volume of the universe; actual black hole radius; actual length contraction amount and its reason; actual mass increase amounts of moving bodies; free energy generating methods such and such.

I shall not demand a patent right. You can use freely above stated things without asking.

\section{References}

1. Kavak M. 2018, Complementary Inferences on Theoretical Physics and Mathematics, OSF Preprints, Available online: https:/osf.io/tw52w/

2. Kavak M. 2016, On the Uncertainty Principle, American Journal of Physics and Applications, Vol. 4, No. 4, 2016, pp. 90-123. Available online: https://osf.io/t8zqw/ 